\title{
The Field Algebra and Its Positive Linear Functionals
}

\author{
W. WYSS ${ }^{\star}$ \\ Department of Mathematics and Physics, University of Colorado, Boulder, Colorado
}

Received February 11, 1972

\begin{abstract}
We show that positive linear functionals on the field algebra are necessarily continuous and can be represented by conical measures. Furthermore extension theorems for continuous linear functionals, defined on a subspace of the field algebra, to positive linear functionals are discussed.
\end{abstract}

\section{Introduction}

It is well known [1] that Wightman's axiomatic theory of quantized fields can be discussed in terms of a topological *-algebra $\mathfrak{A}$, called the field algebra, and its positive linear functionals that vanish on a certain subspace. The study of continuous linear functionals vanishing on this subspace is the content of the so called linear program in Quantum Field Theory. Our intention here is to learn as much as possible about positive continuous linear functionals on $\mathfrak{A}$. In Section 2 we study the field algebra, its hermitean part and the positive cone $K$. In Section 3 we find that a positive linear functional on $\mathfrak{A}$ is always continuous. Hence the continuity requirement in Wightman's axioms can be dropped. Section 4 shows that positive functionals are related to conical measures. In Section 5 we are interested in the following situation: given a continuous linear functional $T$ on a closed subspace $M \subset \mathfrak{A}$ and positive on $K \cap M$. Furthermore let $N$ be another closed subspace of $\mathfrak{A}$. Under what conditions does there exist an extension of $T$ to a positive linear functional on $\mathfrak{A}$, vanishing on $N$ ? We have necessary and sufficient conditions for this situation. Section 6 deals with some applications to Wightman's field theory.

\section{The Field Algebra $\mathfrak{P}$, its Hermitean Part $\mathfrak{H}_{0}$ and the Positive Cone $K$}

For our purposes the field algebra $\mathfrak{A}$ is modeled over $\subseteq\left(\mathbb{R}^{4 n}\right)$, the Laurent Schwartz test function space [2], as follows

* Supported in part by National Science Foundation, GP 19479, and a Summer Research Initiation Fellowship from the University of Colorado. 
Definition 2.1. Let $\Xi_{0} \equiv \mathbb{C}, \Xi_{n} \equiv \Xi\left(\mathbb{R}^{4 n}\right)$ and $\mathfrak{A}=\bigoplus_{n=0}^{\infty} \Xi_{n}$, the topological direct sum of the locally convex topological vector spaces $\Xi_{n}$. An element of $\mathfrak{U}$ thus is a terminating sequence

$$
f=\left\{f_{0}, f_{1}, \ldots\right\}, \quad f_{n} \in \Xi_{n} .
$$

Now we equip $\mathfrak{A}$ with the multiplication

and the involution

$$
\begin{gathered}
(f g)_{n}\left(x_{1}, \ldots, x_{n}\right)=\sum_{k=0}^{n} f_{k}\left(x_{1}, \ldots, x_{k}\right) \\
g_{n-k}\left(x_{k+1}, \ldots, x_{n}\right),
\end{gathered}
$$

$$
(f *)_{n}\left(x_{1}, \ldots, x_{n}\right)=\overline{f\left(x_{n}, \ldots, x_{1}\right)} .
$$

Thus $\mathfrak{H}$ becomes a topological $*$-algebra with the identity $1=\{1,0,0, \ldots\}$; we call it the field algebra.

Definition 2.2. The hermitean part $\mathfrak{U}_{0}$ of the field algebra $\mathfrak{A}$ is given by $\mathfrak{R}_{0}=\left\{f \in \mathfrak{U} ; f^{*}=f\right\}$.

Definition 2.3. Let $\pi=\left\{f \in \mathfrak{I} ; f=\sum_{i} \lambda_{i}\left(g^{i}\right)^{*}\left(g^{i}\right), \lambda_{i} \geqq 0, g^{i} \in \mathfrak{A}\right\}$, where the sum extends over finitely many terms only. Furthermore call $K=\bar{\pi}$ the positive cone of $\mathfrak{U}$.

Lemma 2.1. a) $\mathfrak{U}_{0}$ is a real complete locally convex topological vector space:

b) $K$ is a closed proper cone in $\mathfrak{U}_{0}$ and is generating, i.e., $\mathfrak{A}_{0}=K-K$.

c) K turns $\mathfrak{H}_{0}$ into an ordered topological vector space.

Proof. a) From the general theory of locally convex tropological vector spaces and the fact that the involution * is continuous it follows that $\mathfrak{U}_{0}$ is complete under the induced topology. Note that $\mathfrak{A}_{0}$ is not an algebra under the multiplication in $\mathfrak{H}$, but only a real vector space.

b) First we show that $\pi$ is proper and generating. Suppose that $p \in \pi$ and $-p \in \pi$, i.e. $p=\sum_{i} \lambda_{i}\left(f^{i}\right)^{*} f^{i}$ and $-p=\sum_{i} \mu_{j}\left(g^{i}\right)^{*} g^{i}$. In components these statements read

$$
\begin{gathered}
p_{n}\left(x_{1}, \ldots, x_{n}\right)=\sum_{i} \lambda_{i} \sum_{k=0}^{n} \overline{\left(f^{i}\right)_{k}\left(x_{k}, \ldots, x_{1}\right)} \\
\left(f^{i}\right)_{n-k}\left(x_{k+1}, \ldots, x_{n}\right) \\
(-p)_{n}\left(x_{1}, \ldots, x_{n}\right)=\sum_{i} \mu_{j} \sum_{l=0}^{n} \overline{\left(g^{j}\right)_{l}\left(x_{l}, \ldots, x_{1}\right)} \\
\left(g^{j}\right)_{n-l}\left(x_{l+1}, \ldots, x_{n}\right) .
\end{gathered}
$$


Starting the comparison from the zero's component upwards gives us

$$
\begin{gathered}
p_{0}=\sum_{i} \lambda_{i} \overline{\left(f^{i}\right)_{0}}\left(f^{i}\right)_{0} \geqq 0, \\
(-p)_{0}=-p_{0}=\sum_{i} \mu_{j} \overline{\left(g^{j}\right)_{0}}\left(g^{j}\right)_{0} \geqq 0 .
\end{gathered}
$$

Hence $p_{0}=0$, or $\left(f^{i}\right)_{0}=0 \forall i$, and $\left(g^{i}\right)_{0}=0, \forall j$.

It then follows that also $p_{1}=0$. Furthermore

$$
\begin{gathered}
p_{2}\left(x_{1}, x_{2}\right)=\sum_{i} \lambda_{i} \overline{\left(f^{i}\right)_{1}\left(x_{1}\right)}\left(f^{i}\right)_{1}\left(x_{2}\right) \\
(-p)_{2}\left(x_{1}, x_{2}\right)=-p_{2}\left(x_{1}, x_{2}\right)=\sum_{i} \mu_{i} \overline{\left(g^{i}\right)_{1}\left(x_{1}\right)}\left(g^{i}\right)_{1}\left(x_{2}\right)
\end{gathered}
$$

or on the diagonal $x_{1}=x_{2}$ we get

$$
\begin{aligned}
& p_{2}\left(x_{1}, x_{1}\right)==\sum_{i} \lambda_{i}\left|\left(f^{i}\right)_{1}\left(x_{1}\right)\right|^{2} \geqq 0, \\
& -p_{2}\left(x_{1}, x_{1}\right)=\sum_{i} \mu_{l}\left|\left(g^{i}\right)_{1}\left(x_{1}\right)\right|^{2} \geqq 0
\end{aligned}
$$

implying that $\left(f^{i}\right)_{1}\left(x_{1}\right)=0 \forall i$, and $\left(g^{i}\right)_{1}\left(x_{1}\right)=0, \forall j$ and thus $p_{2}\left(x_{1}, x_{2}\right)=0$ and also $p_{3}=0$. Continuing this way we see that $p \in \pi$ and $-p \in \pi$ imply $p=0$, i.e. $\pi$ is proper.

That $\pi$ is generating follows from the decomposition of $f \in \mathfrak{A}_{0}$ by

$$
f=\frac{1}{4}(1+f)(1+f)-\frac{1}{4}(1-f)(1-f) .
$$

Since $\pi$ is generating, $K=\bar{\pi}$ has also the generating property.

It only remains to show that $K$ is proper. Suppose that $p \in K$ and $-p \in K$, i.e. $p=\lim _{\alpha} p_{\alpha},-p=\lim _{\beta} q_{\beta}$, where $p_{\alpha}, q_{\beta} \in \pi$. The same reflections as for $\pi$ lead us to the desired result.

c) The cone $K$ determines an order relation [4] in $\mathfrak{A}_{0}$ by

$$
f<g \Leftrightarrow g-f \in K .
$$

The field algebra $\mathfrak{A}$ is thus decomposed into $\mathfrak{A}=\mathfrak{A}_{0} \oplus i \mathfrak{U}_{0}$ where $\mathfrak{A}_{0}$ is ordered by the generating cone $K$. The field algebra itself has some nice algebraic and topological properties [3].

Lemma 2.2. a) $\mathfrak{A}$ has no divisors of zero.

b) The center of $\mathfrak{A}$ is $\mathbb{C}$ and no element outside the center has an inverse.

c) 0 and 1 are the only idempotents.

d) $\mathfrak{P}$ has no minimal ideals.

e) $\mathfrak{U}$ is semisimple. 
Proof. a) The condition $f \cdot g=0, f \neq 0$ reads in components

$$
\begin{gathered}
(f g)_{n}\left(x_{1}, \ldots, x_{n}\right)=\sum_{k=0}^{n} f_{k}\left(x_{1}, \ldots, x_{k}\right) \\
g_{n-k}\left(x_{k+1}, \ldots, x_{n}\right)=0 \forall n,
\end{gathered}
$$

from which, staring at $n=0$, one easily deduces that $g=0$.

b) The center of $\mathfrak{i l}$ consist of all elements $f$ such that $f g=g f$, $\forall g \in \mathfrak{Q}$. In components this equation leads to $f=\lambda \cdot 1$. If then $f \notin \mathbb{C}$, which means that at least one component $f_{k}\left(x_{1}, \ldots, x_{k}\right) \neq 0, k \neq 0$, the condition $f g=1$ can be solved for $g$ componentwise, but $g$ is never a terminating sequence, i.e. $g \notin \mathfrak{A}$.

c) The equation $f \cdot f=f$ has only two solutions, namely $f=0$ and $f=1$.

d) Suppose $I$ is a minimal left ideal in $\mathfrak{I}$. For $f \in I, f \neq 0$ we have If $\subset I$, but the minimality requires $I f=I$. Hence there is an element $g \in I$ with $g f=f$. This only holds if $g=1$. Since $I$ is proper, $1 \notin I$, leading to a contradiction.

c) The semisimplicity says that the intersection of all maximal left ideals in $\mathfrak{Q}$ is $\{0\}$, or equivalently [5] that

$$
R=\left\{g \in \mathfrak{I} ; \exists(1+f g)^{-1}, \forall f \in \mathfrak{N}\right\}=\{0\} .
$$

But this condition is satisfied because the inverse only exists for non zero elements in the center.

Lemma 2.3. a) $\mathfrak{A}$ is a separable LF-space and thus is complete.

b) $\mathfrak{A}$ is nuclear, barreled, bornological and reflexive

Proof. See [3].

We now address our attention to the hermitean part $\mathfrak{i}_{0}$ of the field algebra. There the positive cone has some interesting properties.

Lemma 2.4. $K$ has no interior point.

Proof. First $\pi$ has no interior points because every neighborhood $U$ of an element $f \in \pi$ contains elements with an odd heighest component; an element $f \in \pi$ however, has an even highest component. Then $K$ has also no interior points. Some more properties of $K$ will be found but we need to know something about $\mathfrak{Q}(\mathfrak{Q}, \mathfrak{2})$, the space of continuous linear transformations from $\mathfrak{A}$ into $\mathfrak{A}[4]$.

Lemma 2.5. Denote by $\mathfrak{B}$ the family of all bounded sets in $\mathfrak{A}$. Then with $B_{1}, B_{2} \in \mathfrak{B}$, the product $B_{1} B_{2}$ is also bounded.

Proof. Let $f \cdot g=L_{f}(g)=R_{g}(f), f, g \in \mathfrak{U}$. 
From the separate continuity of the multiplication in $\mathfrak{Q}$ it follows that $R_{g}(B)$ is bounded, $\forall g \in \mathfrak{A}, \forall B \in \mathfrak{B}$.

Hence the set $\left\{L_{f} ; f \in B, B \in \mathfrak{B}\right\} \subset \mathfrak{Q}(\mathfrak{U}, \mathfrak{I})$ is simply bounded, i.e. $\left\{L_{f}(g) ; f \in B\right\}$ is bounded for any $g$. But $\mathfrak{A}$ is barreled and thus $\left\{L_{f}, f \in B\right\}$ is equicontinuous. It follows now that $\left\{L_{f}(g) ; f \in B_{1}, g \in B_{2}\right\}$ is bounded in $\mathfrak{A}$.

Definition 2.4. Let $\mathfrak{B}_{0}$ be the family of bounded sets in $\mathfrak{A}_{0}$, and $B_{K}=B \cap K-B \cap K, B \in \mathfrak{B}_{0} . K$ is called a strict $\mathfrak{B}$-cone if $\left\{B_{K} ; B \in \mathfrak{B}_{0}\right\}$ is a fundamental subfamily of $\mathfrak{B}_{0}$, i.e. every element of $\mathfrak{B}_{0}$ is contained in a suitable member of $\left\{B_{K} ; B \in \mathfrak{B}_{0}\right\}$.

Lemma 2.6. $K$ is a complete strict $\mathfrak{B}$-cone in $\mathfrak{A}_{0}$.

Proof. First $K$ is complete because $\mathfrak{Q}_{0}$ is complete and $K$ is closed. Now let $B \in \mathfrak{B}_{0}$. From the fact that $\mathfrak{P}_{0}=K-K$ we know that there is a $B_{1} \in \mathfrak{B}_{0}$ such that

$$
B \subset B_{1} \cap K-B_{1} \cap K .
$$

The last property of $K$ will lead us to an interesting result about positive linear functionals on $\mathfrak{A}$.

\section{Positive Linear Functionals on $\mathfrak{P}$ are Continuous}

Definition 3.1. a) Let $\mathfrak{U}^{\prime}=\mathfrak{Q}(\mathfrak{H}, \mathbb{C})$ stand for the locally convex topological vector space of the continuous linear functionals on $\mathfrak{\mathfrak { r }}$.

b) A continuous linear functional $T \in \mathfrak{Q}^{\prime}$ is called hermitean if $T\left(f^{*}\right)=\overline{T(f)}, \forall f \in \mathfrak{A}$.

c) A continuous linear functional $T \in \mathfrak{A}^{\prime}$ is called positive if $T(f) \geqq 0 \forall f \in K$.

d) A positive continuous linear functional $T \in \mathfrak{Y}^{\prime}$ is called a state on $\mathfrak{I}$ provided $T(1)=1$.

Lemma 3.1. a) A positive continuous linear functional is hermitean.

b) A positive continuous linear functional satisfies the inequality (Cauchy-Schwarz inequality)

$$
\left|T\left(f^{*} g\right)\right|^{2} \leqq T\left(f^{*} f\right) T\left(g^{*} g\right) .
$$

c) For a positive continuous linear functional, the condition $T(1)=0$ is equivalent to $T \equiv 0$.

Proof. a) and b) are well known facts [5].

c) From the Cauchy-Schwarz inequality we get

$$
|T(f)|^{2} \leqq T(1) T(f * f) .
$$


Thus $T(1)=0$ implies $T(f)=0 \forall f \in \mathfrak{A}$.

Remark 3.1. Denote by $\mathfrak{I}_{H}(\mathfrak{A}, \mathbb{C})$ the set hermitean continues linear functionals on $\mathfrak{A}$. This is a real locally convex topological vector space. Furthermore let $\mathfrak{I}_{0}^{\prime}=\mathfrak{Q}\left(\mathfrak{H}_{0}, \mathbb{R}\right)$. It is well known that $\mathfrak{U}_{0}^{\prime}$ and $\mathfrak{I}_{H}(\mathfrak{I}, \mathbb{C})$ are isomorphic. The isomorphism $\alpha: \mathfrak{I}_{0}^{\prime} \rightarrow \mathfrak{Q}_{H}(\mathfrak{I}, \mathbb{C})$ is given by

where

$$
(\alpha T)(f)=T\left(f_{1}\right)+i T\left(f_{2}\right),
$$

and

$$
f_{1}=\frac{1}{2}\left(f+f^{*}\right) \quad \text { and } \quad f_{2}=\frac{1}{2 i}\left(f-f^{*}\right)
$$

$$
\left(\alpha^{-1} L\right)(f)=L(f), \quad \forall f \in \mathfrak{A}_{0} .
$$

Thus the positive continuous linear finctionals on $\mathfrak{A}$ correspond in a one-to-one way to elements of

$$
K^{\prime}=\left\{T \in \mathfrak{A}_{0}^{\prime} ; T(f) \geqq 0 \forall f \in K\right\} .
$$

$K^{\prime}$ is called the dual cone of $K \subset \mathfrak{A}_{0} ;$ it turns $\mathfrak{A}_{0}^{\prime}$ into an ordered topological vector space.

We want to recall some topological properties of $\mathfrak{U}^{\prime}$ [3].

Lemma 3.2. a) $\mathfrak{U}=\prod_{n=0}^{\infty}\left(\mathfrak{\Xi}_{n}\right)^{\prime}$.

b) $\mathfrak{A}^{\prime}$ is nuclear and complete.

c) $\mathfrak{U}^{\prime}$ is bornological.

Theorem 3.1. Every positive linear functional on $\mathfrak{A}_{0}$ is continuous.

Proof. We know that $\mathfrak{A}_{0}$ is bornological and $K$ a complete strict $\mathfrak{B}$-cone. This is sufficient to insure the continuity of every positive linear functional on $\mathfrak{A}_{0}$ [4]. The dual cone $K^{\prime} \subset \mathfrak{P}_{0}^{\prime}$ also has some nice properties.

Definition 3.2. Let $E$ be an ordered topological vectorspace and $C$ the corresponding cone.

a) $A$ set of the form $\{x \in E ; a \leqq x \leqq b\}$ is called an orderinterval and is denoted by $[a, b]$.

b) Let $A$ be any subset of $E$ and

$$
[A]=(A+C) \cap(A-C)=\cup\{[f, g] ; f, g \in A\} .
$$

If $A=[A]$, then $A$ is said to be $C$-saturated or $A$ is called a diamond.

c) The cone $C$ is called normal if these exist a basis of neighborhoods of zero consisting of diamonds.

Lemma 3.3. $K^{\prime}$ is a normal cone in $\mathfrak{X}_{0}^{\prime}$. 
Proof. Since $K$ is a strict $\mathfrak{B}$-cone in $\mathfrak{A}_{0}$ and $\mathfrak{A}_{0}^{\prime}$ is bornological, we conclude that $K^{\prime}$ is normal [4].

Lemma 3.4. $K^{\prime}$ is weakly complete and proper in $\mathfrak{X}_{0}^{\prime}$.

Proof. Since every positive linear functional is continuous, the algebraic dual $K^{*}$ coincides with $K^{\prime} . K^{*}$ however is closed in $\mathfrak{U}_{0}^{*}$, the algebraic dual of $\mathfrak{A}_{0}$, and since $\mathfrak{A}_{0}^{*}$ is weakly complete $K^{*}$ is also weakly complete.

$K^{\prime}$ as we know is normal and hence proper.

This lemma enables us to represent positive linear functionals by conical measures.

\section{Positive Functionals on $\mathfrak{A}$ are Represented by Conical Measures}

We apply Choquet's theorem on weakly complete cones and conical measures [6] to $K^{\prime}$.

Definition 4.1. We consider the space $\mathfrak{A}_{0}^{\prime}$ and its topological dual $\mathfrak{U}_{0}^{\prime \prime}=\mathfrak{U}_{0}$.

a) Denote by $\mathfrak{F}$ the family of finite subsets of $\mathfrak{A}_{0}$. For $F \in \mathfrak{F}$ and $T \in \mathfrak{P}_{0}^{\prime}$ let

and

$$
(\sup F)(T)=\sup \{T(f), f \in F\}
$$

$$
\begin{gathered}
H\left(\mathfrak{H}_{0}^{\prime}\right)=\left\{G: \mathfrak{A}_{0}^{\prime} \rightarrow \mathbb{R} ; \text { such that } \exists F_{1}, F_{2} \in \mathfrak{F}\right. \text { with } \\
\left.G(T)=\left(\sup F_{1}\right)(T)-\left(\sup F_{2}\right)(T), \forall T \in \mathfrak{A}_{0}^{\prime}\right\} .
\end{gathered}
$$

Hence $\mathfrak{I}_{0}=\left(\mathfrak{Q}_{0}^{\prime}\right)^{\prime} \subset H\left(\mathfrak{U}_{0}^{\prime}\right)$ and $H\left(\mathfrak{H}_{0}^{\prime}\right)$ is a vectorlattice ordered by $G \geqq 0 \Leftrightarrow G(T) \geqq 0, \forall T \in \mathfrak{U}_{0}^{\prime} . H\left(\mathfrak{V}_{0}^{\prime}\right)$ coincides with the piecewise linear functional on $\mathfrak{X}_{0}^{\prime}$.

b) The positive linear functionals on $H\left(\mathfrak{Q}_{0}^{\prime}\right)$ are denoted by $M^{+}\left(\mathfrak{A}_{0}^{\prime}\right)=\left(H\left(\mathfrak{A}_{0}^{\prime}\right)^{*}\right)^{+}$, and are called positive conical measures on $\mathfrak{I}_{0}^{\prime}$. On $M^{+}\left(\mathfrak{U}_{0}^{\prime}\right)$ we put the weak topology. Furthermore let $M\left(\mathfrak{U}_{0}^{\prime}\right)=M^{+}\left(\mathfrak{U}_{0}^{\prime}\right)$ $-M^{+}\left(\mathfrak{A}_{0}^{\prime}\right)$; this is a complete lattice.

c) $K^{\prime} \subset \mathfrak{U}_{0}^{\prime}$ is closed. A conical measure $\mu \in M\left(\mathfrak{U}_{0}^{\prime}\right)$ is said to be carried by $K^{\prime}$ if

$$
G \in H\left(\mathfrak{U}_{0}^{\prime}\right) \quad \text { and } \quad G=0 \quad \text { on } \quad K^{\prime} \Rightarrow \mu(G)=0 .
$$

d) Let $\mu \in M^{+}\left(\mathfrak{U}_{0}^{\prime}\right)$. We say that $T \in \mathfrak{P}_{0}^{\prime}$ is the resultant of $\mu$ if $\mu(f)=T(f) \forall f \in \mathfrak{U}_{0}=\left(\mathfrak{U}_{0}^{\prime}\right)^{\prime}$.

e) We introduce a partial ordering on $M^{+}\left(\mathfrak{A}_{0}^{\prime}\right)$ as follows: Let $S_{H}=\{\sup F ; F \in \mathfrak{F}\}$ and $\mu, v \in M^{+}\left(\mathfrak{A}_{0}^{\prime}\right)$, then

$$
\mu<v \Leftrightarrow \mu(G) \leqq v(G), \quad \forall G \in S_{I I} .
$$


Theorem 4.1. Let $T \in K^{\prime} \subset \mathfrak{U}_{0}^{\prime}$. Then there exists a maximal conical measure $\mu \in M^{+}\left(\mathfrak{U}_{0}^{\prime}\right)$ such that $\mu$ is carried by $K^{\prime}$ and has resultant $T$, i.e. $T(f)=\mu(f), \forall f \in \mathfrak{U}_{0}$.

Proof. Since $K^{\prime}$ is a weakly complete, convex proper cone, we can apply Choquet's theorem.

Remark 4.1. It is well known [7] that positive linear functionals on a $C^{*}$-algebra are continuous and can be represented by Radon measures. For the field algebra we have a similar situation except that we have to deal with conical measures. This difference is reflected in the fact that a $C^{*}$-algebra corresponds to bounded operators on some Hilbert space whereas the field algebra corresponds to unbounded operators on some Hilbert space; the correspondence is given by the Gel'fand-Segal construction.

\section{Extension Theorems}

The goal of this section is to establish conditions under which a continuous linear functional defined on a subspace $M \subset \mathfrak{P}_{0}$ and positive on $K \cap M$ can be extended to a positive linear functional on $\mathfrak{U}_{0}$ in such a way that a given subspace $L \subset \mathfrak{A}_{0}$ belongs to the kernel of the extension. We will break up the discussion into two parts. First we investigate the extensions from a given subspace $M$ and secondly we look for those extensions vanishing on a given subspace.

All the following discussion are based on the Hahn-Banach theorem, a consequence of which is the bipolar theorem.

Lemma 5.1. (Bipolar theorem). Let $A \subset \mathfrak{U}_{0}$ be any subset. $A^{0}=\left\{T \in \mathfrak{U}_{0}^{\prime} ; T(f) \leqq 1, \forall f \in A\right\}$ is called the polar of $A$. Then $A^{00}$ is the weakly closed, convex hull of $A \cup\{0\}$.

Proof. See (4).

Lemma 5.2. Let $N$ be a closed subspace of $\mathfrak{A}_{0}$, then $(K \cup N)^{00}=\overline{K+N}$, or equivalently $\overline{(K+N)^{0}}=(K \cup N)^{0}$.

Proof. By the bipolar theorem $(K \cup N)^{00}$ is equal to the weak closure of the convex hull of $K$ and $N$. But the closure of a convex set is the same for all admissable topologies.

We are now in a position to prove a key lemma.

Lemma 5.3. $\overline{K+\mathbb{R} \cdot 1}=\mathfrak{U}_{0}$.

Proof. Since there is only the trivial positive functional vanishing on 1, we have $K^{\prime} \cap(\mathbb{R} \cdot 1)^{0}=\{0\}$. But $\left(K^{\prime}\right)^{0}=-K$ and hence by the above lemma

$$
\text { conv. closure }(-K \cup \mathbb{R} \cdot 1)=\mathfrak{U}_{0} \text {, }
$$


or

equivalently

$$
\begin{aligned}
& \overline{-K+\mathbb{R} \cdot 1}=\mathfrak{U}_{0} ; \\
& \overline{K+\mathbb{R} \cdot 1}=\mathfrak{U}_{0} .
\end{aligned}
$$

Let now $M$ be any closed subspace of $\mathfrak{Y}_{0}$ and $T$ a nontrivial continuous linear functional defined on $M$ and positive on $K \cap M$. The kernel of $T$ in $M$ is denoted by $\operatorname{ker} T$.

Theorem 5.1. There exists a positive linear extension of $T$, denoted by ext $T$, to $\mathfrak{U}_{0}$, provided $K \cap M \nmid \operatorname{ker} T$, iff

$$
\overline{K+\operatorname{ker} T} \neq \overline{K+M} \text {. }
$$

Proof. a) Suppose that $\overline{K+\operatorname{ker} T}=\overline{K+M}$, then by applying the polar operation

or equivalently

$$
\begin{aligned}
-K^{\prime} \cap(\operatorname{ker} T)^{0} & =-K^{\prime} \cap M^{0}, \\
K^{\prime} \cap(\operatorname{ker} T)^{0} & =K^{\prime} \cap M^{0} .
\end{aligned}
$$

Thus every positive linear functional, vanishing on $\operatorname{ker} T$ also vanishes on $M$ and hence its restriction to $M$ is trivial, contrary to our assumption.

b) If $\overline{K+\operatorname{ker} T} \neq \overline{K+M}$, then $\overline{K+\operatorname{ker} T} \subseteq \overline{K+M}$ and thus $-K^{\prime} \cap M^{0} \subsetneq-K^{\prime} \cap(\operatorname{ker} T)^{0}$ or $K^{\prime} \cap M^{0} \subsetneq K^{\prime} \cap(\operatorname{ker} T)^{0}$. Hence there exists a positive linear functional vanishing on $\operatorname{ker} T$ and not vanishing on $M$, meaning that there exists an extention of $T$.

Corollary 5.1. Under the same assumptions as above plus the requirement that $1 \in M$, there exists an extension of $T$ iff

or equivalently

$$
\overline{K+\operatorname{ker} T} \neq \mathfrak{Q}_{0}
$$

$$
-1 \notin \overline{K+\operatorname{ker} T} \text {. }
$$

Proof. The first part follows from the previous theorem, because $\overline{K+M}=\mathfrak{V I}_{0}$. Secondly if $-1 \in \overline{K+\operatorname{ker} T}$ then $\overline{K+\operatorname{ker} T}$ and $K+\mathbb{R}$ $C \overline{K+\operatorname{ker} T}$ or taking the closure we get that $K+\operatorname{ker} T=\mathfrak{P}_{0}$, meaning that there is no extension.

Theorem 5.2. [8]. Let $T$ be a continuous linear functional defined on $M=\{\lambda p ; \lambda \in \mathbb{R}, p \in K\}$ and positive on $K \cap M$. Then there always exist an extension of $T$.

Proof. The condition for the extension theorem are satisfied, because

$$
\overline{K+\operatorname{ker} T}=K \neq \overline{K+M} \text {, since } K \text { is proper . }
$$

Corollary 5.2. $\mathfrak{H}$ is *-semisimple in the sense that the intersection of all left kernels for positive linear functionals is zero, i.e.

$$
\bigcap_{T \in K^{\prime}}\left\{f ; T\left(f^{*} f\right)=0\right\}=0 \text {. }
$$


Proof. Assume $f \neq 0$ and $f \in \bigcap_{T \in K^{\prime}}\left\{g ; T\left(g^{*} g\right)=0\right\}$. Then $T\left(f^{*} f\right)$ $=0 \forall T \in K^{\prime}$. But for an element $f^{*} f \in K$ there always exists a positive linear functional $T \in K^{\prime}$ such that $T\left(f^{*} f\right)>0$, and thus $f=0$.

\section{Corollary 5.3. $\bigcap_{T \in K^{\prime}} \operatorname{ker} T=0$.}

Proof. An element $f \in \mathfrak{U}$ satisfies the property $T\left(f^{*} f\right)=0 \forall T \in K^{\prime}$ iff $T(f)=0 \forall T \in K^{\prime}$. The necessity of this statement follows from the Cauchy-Schwarz inequality $|T(f)|^{2} \leqq T(1) T\left(f^{*} f\right)$. Conversely assume that $f$ satisfies $T(f)=0 \forall T \in K^{\prime}$. Then $T_{g}(f) \equiv T\left((\lambda+g) f(\lambda+g)^{*}\right)$ is a positive linear functional if $T \in K^{\prime}$, and thus $T\left((\lambda+g) f(\lambda+g)^{*}\right)=0$ $\forall \lambda \in \mathbb{C}, g \in \mathfrak{A}$. Setting $\lambda=1,-1, i,-i$, one concludes that $T\left(f^{*} f\right)=0$ $\forall T \in K^{\prime}$.

Having an extension theorem, we are next concerned with the existence of positive linear functionals vanishing on a given closed subspace $N \subset \mathfrak{Q H}_{0}$.

Theorem 5.3. Let $N$ be a closed subspace of $\mathfrak{A}_{0}$. Then there exists a nontrivial positive linear functional $T$ on $\mathfrak{I}_{0}$, such that $N \subset \operatorname{ker} T, K \Varangle \operatorname{ker} T$, iff

$$
\overline{K+N} \neq \mathfrak{Q}_{0} \text {. }
$$

Proof. a) Assume that $\overline{K+N}=\mathfrak{U}_{0}$, then $-K^{\prime} \cap N^{0}=0$, i.e. only the trivial positive functional can vanish on $N$.

b) If $\overline{K+N} \subsetneq \mathfrak{H}_{0}$, then $-K^{\prime} \cap N^{0} \subsetneq\{0\}$ and thus there exists a positive linear functional vanishing on $N$. Combining this theorem with the extension theorem, we get the following statement.

Theorem 5.4. Let $M$ and $N$ be closed subspaces of $\mathfrak{U}_{0}$ such that $1 \in M$. Suppose that $T$ is a continuous linear functional defined on $M$, positive on $K \cap M$ and $T(1)=1$. Furthermore assume that $N \cap M \subset \operatorname{ker} T$. If there exists an extension of $T$ to a positive linear functional on $\mathfrak{A}_{0}$, then the set of extensions contains positive linear functionals vanishing on $N$, iff $-1 \notin \overline{K(T)+N}$, where $K(T)=\overline{K+\operatorname{ker} T}$.

Proof. The necessary and sufficient condition for the existence of an extension of $T$ is given by $K(T) \neq \overline{K+M}$. Note then that a continuous linear functional, positive on $K(T)$, is an extension of $T$, up to normalization. Since $-1 \notin \overline{K(T)+N}$, we have that $\overline{K(T)+N} \neq \mathfrak{U}_{0}$. Hence there always exists a continuous linear functional positive on $K(T)$.

Theorem 5.5. If $M$ is finite dimensional, $1 \in M, K \cap \operatorname{ker} T=\{0\}$ and $T(1)=1$. Then there always exists an extension of $T$ to a positive linear functional.

Proof. Under the assumption that $\operatorname{ker} T$ is finite dimensional and $K \cap \operatorname{ker} T=\{0\}$, we know [9] that $K+\operatorname{ker} T$ is closed and thus $-1 \notin \overline{K+\operatorname{ker} T}=K+\operatorname{ker} T$. 
Corollary 5.4. Let $p \in K, p \neq \lambda \cdot 1, \lambda>0$, and $M=\{\lambda+\mu p ; \lambda, \mu \in \mathbb{R}\}$. Define $T$ on $M$ by $T(1)=1, T(p)>p_{0}$, where $p_{0}$ is the zero component of $p$. Then there exists a positive functional ext $T$ such that $\left.\operatorname{ext} T\right|_{M}=T$.

Proof. The kernel of $T$ is given by $\operatorname{ker} T=\mathbb{R}(T(p)-p)$. Because $T(p)>p_{0}$ it follows that $K \cap \operatorname{ker} T=\{0\}$ and hence Theorem 5.5 applies.

The results of Section 5 hold generally for locally convex *-algebras with identity provided one restricts attention to continuous positive linear functionals.

\section{Implications for Wightman's Axiomatic Theory of Quantized Fields}

A Wightman theory for a neutral scalar field [1] is given by a positive linear functional on $\mathfrak{U}$, vanishing on a closed subspace $L \subset \mathfrak{U}_{0}$. This subspace $L$ represents the linear program in quantum field theory and will be defined below.

Definition 6.1. a) Let $F: \mathfrak{U} \rightarrow \mathfrak{U}$ be the Fourier transform defined by

$$
\begin{gathered}
(F f)_{n}\left(x_{1}, \ldots x_{n}\right)=(2 \pi)^{-2 n} \int e^{i\left(p_{1} x_{1}+\cdots+p_{n} x_{n}\right)} f_{n}\left(p_{1}, \ldots, p_{n}\right) d p_{1} \ldots d p_{n} \\
(F f)_{0}=f_{0} .
\end{gathered}
$$

This is an automorphism of $\mathfrak{Y}$.

The left ideal

$$
\begin{aligned}
I_{1}= & \left\{f \in \mathfrak{U} ; f_{0}=0,(F f)_{n}\left(p_{1}, \ldots, p_{n}\right)=0 \quad \text { if } \quad q_{k} \equiv \sum_{l=k}^{n} p_{l} \in \bar{V}_{+},\right. \\
& \forall k, 1 \leqq k \leqq n, \forall n\}
\end{aligned}
$$

is related to the spectrum condition and is called the spectrum ideal.

b) Let $I_{2}$ be the two-sided ideal generated by elements of the form $f_{n}\left(x_{1}, \ldots, x_{n}\right)=\varphi_{n}\left(x_{1}, \ldots, \underline{x}, \ldots, x_{n}\right)-\varphi_{n}\left(x_{1}, \ldots, \underline{x}_{\pi}, \ldots, x_{n}\right), \quad n=1,2, \ldots$, where $\underline{x}=\left\{x_{l}, x_{l+1}, \ldots, x_{l+k}\right\} \subset\left\{x_{1}, \ldots, x_{n}\right\}$ for any $l$ and $k$, and $\pi$ is any permutation of the elements in $\underline{x}$; furthermore $\varphi_{n}\left(x_{1}, \ldots, \underline{x}, \ldots x_{n}\right)=0$ if $\left(x_{i}-x_{j}\right)^{2}>0, \forall x_{i}, x_{j} \in \underline{x}, i \neq j . I_{2}$ is called the locality ideal.

c) Let

$$
\begin{gathered}
((a, \Lambda) f)_{n}\left(x_{1}, \ldots, x_{n}\right)=f\left(\Lambda^{-1}\left(x_{1}-a\right), \ldots, \Lambda^{-1}\left(x_{n}-a\right)\right) \\
((a, \Lambda) f)_{0}=f_{0}
\end{gathered}
$$

be the action of the Poincare group on $\mathfrak{A}$. Then the subspace

$$
I_{3}=\{f \in \mathfrak{P} ; f=g-(a, \Lambda) g ; \forall(a, \Lambda)\}
$$

corresponds to the Poincaré invariance. 
d) Let $N$ be the closed linear space of $I_{1}, I_{2}, I_{3}$.This is a closed linear subspace of $\mathfrak{Q}$ and is called the Wightman kernel. A positive linear functional $W$ on $\mathfrak{A}$ such that $N \subset \operatorname{ker} W$ is called a Wightman functional.

e) $N$ can uniquelly be written as $N=N_{1}+i N_{2}$, where $N_{1}$ and $N_{2}$ are closed linear subspaces of $\mathfrak{U}_{0}$. A positive linear functional on $\mathfrak{U}$, vanishing on $N$, is equivalent to a positive linear functional on $\mathfrak{A}_{0}$, vanishing on the closed linear span $L=L\left(N_{1}, N_{2}\right)$ of $N_{1}$ and $N_{2}$. Hence a Wightman functional is characterized by $W \in K^{\prime}, W(L)=0$. Note that $1 \notin L . L$ is called the hermitean Wightman kernel.

We are now in a position to characterize the existence of Wightman functionals.

Theorem 6.1. Let $M$ be a closed subspace of $\mathfrak{A}_{0}$ with $1 \in M$, and $L$ the hermitean Wightman kernel. A continuous linear functional $T$ defined on $M$ such that $T(K \cap M) \geqq 0, L \cap M \subset \operatorname{ker} T$ and $T(1)=1$ can be extended to a Wightman functional iff $-1 \notin \overline{K(T)+L}$, where $K(T)=\overline{K+\operatorname{ker} T}$.

Proof. This is an immediate consequence of Lemma 5.3 and Theorem 5.4.

Note that the general extension theorem depends on the detailed structure of $\operatorname{ker} T$ and of $L$.

Acknowledgements. We would like to thank Professors H. J. Borchers and A. Ramsay for their interest in this work and for helpful suggestions.

\section{References}

1. Borchers, H. J.: Nuovo Cimento 24, 214 (1962).

2. Schwartz, L.: Theorie des distributions, II. Paris:Hermann 1959.

3. Wyss, W.: Lectures in theoretical physics, Vol. XI. D. New York: Gordon and Breach Interscience Publishers, Boulder 1968.

4. Peressini, A. L.: Ordered topological vector spaces. New York: Harper \& Row 1967. Schaefer, H. H.: Topological vectorspaces. New York: Macmillan Comp. 1966.

5. Neumark, M.A.: Normierte Algebren. Berlin: Deutscher Verlag der Wissenschaften, 1959.

6. Choquet, G.: Lectures on analysis. New York: W. A. Benjamin, Inc. 1969.

7. Dixmier, J.: Les $C^{*}$-algebres et leurs representations. Paris: Gauthier-Villars 1964.

8. Uhlmann, A., Lassner, G.: Commun. math. Phys. 7, 152 (1968).

9. Bourbaki, N.: Espaces vectoriels topologiques 1189 (1953) p. 78, Actualites Scientifiques et Industrielle, No. 1189, Paris, 1953. 